\title{
Sorption behaviors of Cs and Eu onto altered rock formed around fault
}

\author{
YUSUKE WATANABE $^{1}$ AND KoKI KaSHIWAYA ${ }^{2}$ \\ ${ }^{1}$ Japan Atomic Energy Agency, 1-64 Yamanouchi, Akiyo, \\ Mizunami, Gifu, 509-6132, Japan, \\ (watanabe.yusuke79@jaea.go.jp) \\ ${ }^{2}$ Kyoto Univesity, Graduate School of Engineering, Kyoto \\ daigaku-katsura, Nishikyo-ku, Kyoto, 615-8540, Japan \\ ( kashiwaya.koki.8a@kyoto-u.ac.jp)
}

In the safety assessment of geological disposal of radioactive waste, groundwater flow and water-rock interaction are the key processes to understand the migration behavior of radionuclide in the natural barrier system. Fractures in crystalline rock function as a groundwater flow path. In particular, damage zones formed around faults are considered as significant flow path because of having many fractures and their connectivity. The fractures in damage zone contain clay minerals formed by water-rock interaction in fracture surface, and it is possible to have high sorption capacity of radionuclides. In order to understand the sorption capacity and controlling processes of fractures in the damage zone, we conducted sorption experiments of $\mathrm{Cs}$ and $\mathrm{Eu}$, which are representative monovalent and trivalent cations, respectively. The rock sample were collected from boreholes excavated around fault at the $300 \mathrm{~m}$ depth of research gallery at the Mizunami Underground Research Laboratory.

To compare the mineral composition and sorption behavior, we collected rock samples at a different distance from the fault plane, which has various degree of alteration. Clay minerals such as illite and smectite are found in all samples, and contents of them were high at the fault plane and decreased with distance from fault plane. Sorption of Cs onto damage zone rock did not depend on $\mathrm{pH}$ and ionic strength, whereas Eu sorption was depending on them. These results suggest that sorption of $\mathrm{Cs}$ and $\mathrm{Eu}$ are controlled by illite and smectite in the damage zone where Cs strongly sorbed by forming the inner-sphere complex, whereas Eu weakly sorbed by forming the outer-sphere complex. On the other hand, in the protolith rock, Eu sorption was possibly formed inner-sphere complex by sorbing onto biotite. These results suggest that sorption behavior of cation is depending on the mineral composition of rocks which is possibly altered by faulting and subsequent water-rock interaction.

This work has been supported in part by a grant from the Radioactive Waste Management Funding and Research Center. 